\title{
Evaluation of a Vision-based Tactile Sensor
}

\author{
Kazuto Kamiyama, Hiroyuki Kajimoto, Naoki Kawakami and Susumu Tachi \\ Graduate School of Information Science and Technology \\ The University of Tokyo, Tokyo, Japan \\ Email: \{kazuto, kaji, kawakami, tachi\}@ star.t.u-tokyo.ac.jp
}

\begin{abstract}
Humans can perceive not only the magnitude but also the direction of force applied on the fingertip. When we grasp an object, the weight of it is felt through force that is parallel to the skin of the fingertip, which is how the object can be grasped without slipping. Focusing on this point, we have developed a tactile sensor that can measure a distribution of force vectors. The measurement method is as follows. The tactile sensor consists of a transparent elastic body, blue and red markers inside the elastic body, and a color CCD camera. An applied force on the elastic body results in movements of the markers, which are acquired by the CCD camera. The distribution of force vectors is calculated using this information. This paper reports experimental evaluation results concerning accuracy of determining position of markers, determination of magnitude and direction of force, spatial resolution, and calculation timing.
\end{abstract}

\section{INTRODUCTION}

We aquire environmental information through optical, acoustic, tactile sensation and so on. Though it's a well known fact, only tactile sensation has different aspect whereas optical and acoustic sensation aquire the external information. The aspect is to generate consciousness that regions of body such as arm and leg are part of ourselves. Usually, we are not concious of it due to nothing special. However, becoming paralysed on the arm by loss of blood flow, feeling as if the arm were not part of ourselves is arisen.

Just as tactile sensation is important for humans, it is quite useful for robots as well. Robots equipped with a tactile sensor are more capable of fine manipulation and avoiding critical behaviors. Providing tactile sensation to a robotic hand and fingertip is particularly necessary for delicate operation. As an example, suppose we have the simple task of picking up a fragile object on a table. When we execute this operation, the weight of the object is felt not only by contraction of the muscle but also force parallel to the skin of the fingertip. The object is safely picked up by adjusting the proportion of perpendicular and parallel force on the skin. In a similar manner, robots require sensing the force on the fingertip to perform the operation dexterously.

To detect the weight of the object while grasping it, it is necessary to sense the force parallel to the surface of a tactile sensor. This means that the tactile sensor needs to measure distribution of force vectors, but there are few sensors that are able to measure such a thing[1]-[4]. Most of sensors use a sensing unit in which electric resistance or capacitance depends on force. To use such sensors with the intent to measure the distribution of force vectors, the wiring becomes quite complex and the size of the unit increases with the amount of necessary measured information. When the sensors are built into a robot hand, this becomes a severe problem in terms of miniaturizing and high-density sensing

An alternative tactile sensing method makes use of optical information. In particular, sensors using visual images have great potential for decreasing the amount of wiring. There are only a few known sensors that can measure distribution of force vectors[5]. Ohoka et. al developed a method using an elastic sheet and a transparent board parallel to the sheet in which pyramidal protrusions are arranged. An applied force makes the protrusions contact the sheet, and the force is obtained by measuring contact area[6]. Ohoka's method uses four protrusions to acquire a force vector, and is therefore inadequate for high-density sensing. Ferrier et. al proposed a tactile sensor that used markers inside an elastic body and a fiber scope[7]. This sensor is formed into a fingertip shape and is successfully miniaturized. However, only a relatively small amount of information is actually measured, and a complex processing method is used to acquire direction of force, which could potentially lead to inaccuracy and instability.

We focus on sensing methods that use visual images and have proposed a vision-based tactile sensor[8]. This sensor can measure distribution of force vectors, is easily miniaturized, and measures with high density.

\section{THEORY OF MEASURING DISTRIBUTION OF FORCE VECTORS}

The tactile sensor which we have proposed uses a transparent elastic body and a color CCD camera. By photographing a certain marker on the interior of an elastic body by CCD, when force is applied to the surface, the variation information of the interior is measured, and is used to reconstruct a force vector distribution.

Various methods can be considered to acquire variation information. Our current approach is to measure the horizontal movement of spherical markers in the elastic body, which are located in an $N \times N$ array at a specified depth. To gather sufficient information for the reconstruction of the force vectors, we used two layers of markers that are located at different depths (Fig.1). These layers could be distinguished by the colors of the markers (red and blue). We set the $x-y$ plane parallel to the sensor surface and the $z$ axis extending vertically on the interior. By measuring these markers from a positive $z$ direction with a CCD camera, we could obtain two sets of two-dimensional movement vectors at different depths, 
so the amount of information is increased and the distribution of force vectors can be readily obtained.

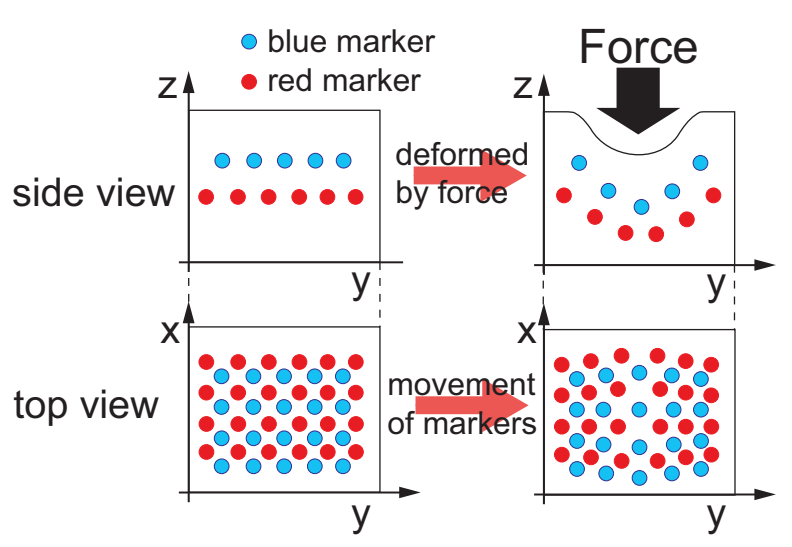

Fig. 1. Overview of Image-based Sensing

\section{A. Measuring the displacements of markers}

To measure the movements of the markers, as preprocessing, the position of markers is determined from the CCD image when the elastic body doesn't vary. This preprocessing allows the markers to be located at arbitrary positions and eliminates the need for camera positioning. To pinpoint the marker center position, a center of mass measurement method is applied. Herewith, the center position, which was originally an integer value based on a pixel unit, becomes a real number with subpixel accuracy.

When force is applied to the elastic body surface, the markers will move, and this movement is observed by the CCD. The picture is subdivided into smaller squares, each of which is centered on the marker position measured in the initial state that force is not applied. By taking the difference of the position of the center in each square of the picture before and after the movement, the markers' translation in the $x y$-direction can be calculated. If the movement falls outside of the divided square, a so-called 'tracking' method can be applied, which only uses two sequential images.

\section{B. Obtaining distribution of force vectors from displacement vectors}

In order to obtain force vectors from movement vectors, we use the theory of elasticity [9] by assuming that the elastic body is half-spaced, uniform and has linearity. We set the $z$ axis perpendicular to the elastic body's interior surface, and the $x y$-plane parallel to the surface of the elastic body. Following Eq. 2 and Eq. 1, we express the movement vector $\vec{u}=\left(u_{x}, u_{y}\right)$ of the interior point $\vec{r}=(x, y, z)$ within a plane parallel to the $x y$-plane when a force vector $\vec{f}=\left(f_{x}, f_{y}, f_{z}\right)$ is applied to the surface of the elastic body. Here, $\sigma$ is a Poisson ratio, which is set to 0.5 , by assuming that the elastic body is ideally incompressible. $E$ is Young's modulus and must be appropriately defined according to the actual elastic body used, but from this equation it is apparent that $E$ is in effect only multiplying the whole equation by a constant, so it is set to 1 in the sequel.

$$
\begin{aligned}
u_{x}= & \frac{1+\sigma}{2 \pi E}\left\{\left[\frac{x z}{r^{3}}-\frac{(1-2 \sigma) x}{r(r+z)}\right] f_{z}+\frac{2(1-\sigma) r+z}{r(r+z)} f_{x}\right. \\
& \left.+\frac{\left[2 r(\sigma r+z)+z^{2}\right] x}{r^{3}(r+z)^{2}}\left(x f_{x}+y f_{y}\right)\right\}, \\
u_{y}= & \frac{1+\sigma}{2 \pi E}\left\{\left[\frac{y z}{r^{3}}-\frac{(1-2 \sigma) y}{r(r+z)}\right] f_{z}+\frac{2(1-\sigma) r+z}{r(r+z)} f_{y}\right. \\
& \left.+\frac{\left[2 r(\sigma r+z)+z^{2}\right] y}{r^{3}(r+z)^{2}}\left(x f_{x}+y f_{y}\right)\right\} .
\end{aligned}
$$

From these equations, when an unit force $\vec{f}=\left(f_{x}, f_{y}, f_{z}\right)$ is applied in each direction $x, y, z$, i.e. $(1,0,0),(0,1,0)$, $(0,0,1)$, the displacement vector of the point in the plane at certain depth $z=z_{1}$ is calculated. We represent this vector by $\vec{u}_{f x}=\left(h_{x x 1}, h_{y x 1}\right), \vec{u}_{f y}=\left(h_{x y 1}, h_{y y 1}\right), \vec{u}_{f z}=$ $\left(h_{x z 1}, h_{y z 1}\right)$. These displacement $h$ can be considered as an impulsive response to unit force of each direction from the origin. When the force applied to the surface of the elastic body is reconsidered as a vector distribution expressed as $\vec{f}(x, y)=\left(f_{x}(x, y), f_{y}(x, y), f_{z}(x, y)\right)$, the displacement vector $\vec{m}_{1}(x, y)=\left(m_{x 1}(x, y), m_{y 1}(x, y), m_{z 1}(x, y)\right)$ for each point $(x, y)$ in the plane at a depth $z_{1}$ and is calculated in the form of a convolution, denoted by an asterisk (Eq.3). Note that this calculation utilizes the assumption of linearity.

$$
\begin{aligned}
& m_{x 1}(x, y)=h_{x x 1} * f_{x}+h_{x y 1} * f_{y}+h_{x z 1} * f_{z}, \\
& m_{y 1}(x, y)=h_{y x 1} * f_{x}+h_{y y 1} * f_{y}+h_{y z 1} * f_{z}
\end{aligned}
$$

A discrete form of this calculation can be expressed as a matrix representation. The displacement vectors and force vectors are sampled at $M \times N$ points, and the $x, y, z$ components of the displacement vector are expressed as $M_{x 1}$, $M_{y 1}, M_{z 1}$ in the matrix. Elements from $(1,1)$ to $(M, N)$ are renumbered using one suffix and are reinserted as a vector. Stress vectors are inserted in the same fashion. By doing so, Eq.3 rewritten in matrix form (Eq.4).

$$
\left[\begin{array}{l}
M_{x 1} \\
M_{y 1}
\end{array}\right]=\left[\begin{array}{lll}
H_{x x 1} & H_{x y 1} & H_{x z 1} \\
H_{y x 1} & H_{y y 1} & H_{y z 1}
\end{array}\right]\left[\begin{array}{c}
F_{x} \\
F_{y} \\
F_{z}
\end{array}\right]
$$

Since this equation calculates displacement of an interior point when a force vector distribution is applied to the surface of the elastic body, its inverse becomes a formula which calculates the force vector distribution by given the measured value $M$. This is the measurement principle of this research.

At this point, consider the number of $M$ vector elements on the left side of the equation and the $F$ vector elements on the right side. Since the number of sampling points is $M \times N$, the number of elements of a displacement vector is $M \times N \times 2$ and the number of elements of a force vector is $M \times N \times 3$, there are more unknowns than equations. Thus, the measuring technique is applied to another height by using color information. As 
the impulse response varies according to depth, we obtain the following equation (Eq.5).

$$
\left[\begin{array}{l}
M_{x 1} \\
M_{y 1} \\
M_{x 2} \\
M_{y 2}
\end{array}\right]=\left[\begin{array}{lll}
H_{x x 1} & H_{x y 1} & H_{x z 1} \\
H_{y x 1} & H_{y y 1} & H_{y z 1} \\
H_{x x 2} & H_{x y 2} & H_{x z 2} \\
H_{y x 2} & H_{y y 2} & H_{y z 2}
\end{array}\right]\left[\begin{array}{c}
F_{x} \\
F_{y} \\
F_{z}
\end{array}\right]
$$

Eq. 5 is simply described as $M=H F$. This becomes a situation with more equations than unknowns, so it is easy to calculate a force vector distribution $F$. Since the matrix $H$ is not a square matrix, it does not have an inverse matrix, so force will be calculated using a pseudo-inverse matrix[10].

\section{DeVICE OVERVIEW}

A system diagram of the sensor used for evaluation is shown in Fig.2.

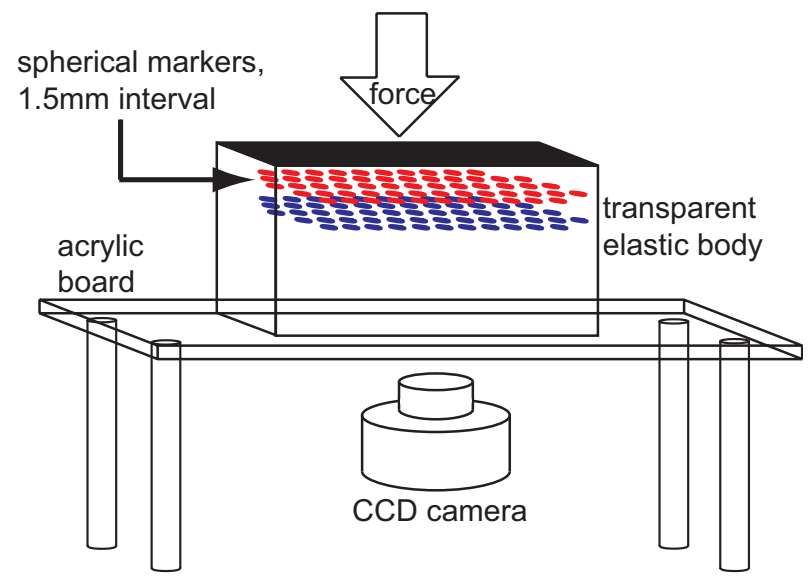

Fig. 2. Image of sensor system

The transparent elastic body has a thickness of $40 \mathrm{~mm}$, a length of $90 \mathrm{~mm}$, a width of $100 \mathrm{~mm}$, and has an opaque black shading layer prepared on its surface. Blue markers are placed at a depth of $3 \mathrm{~mm}$, and red markers at a depth of $6 \mathrm{~mm}$. We use plastic balls (DAICEL FINECHEM, LTD. FREEPLASTIC) with a diameter of $0.6 \mathrm{~mm}$ as color markers, arranged in approximately $1.5 \mathrm{~mm}$ intervals, which is nearly the human's limit of spatial resolution for discriminating between two separated points.

The markers inside the elastic body are photographed through the transparent acrylic board that fixates the elastic body. One pixel corresponds to about a $0.05 \mathrm{~mm}$ square and the size of the photographed area is about $18 \mathrm{~mm} \times 14 \mathrm{~mm}$. The captured image is output in NTSC format and sent to a PC through a capture unit with a USB connection.

Fig. 3 is photographed image by the CCD camera. Blue and Red markers can be observed at a regular interval. Using this image, The force vectors is calculated.

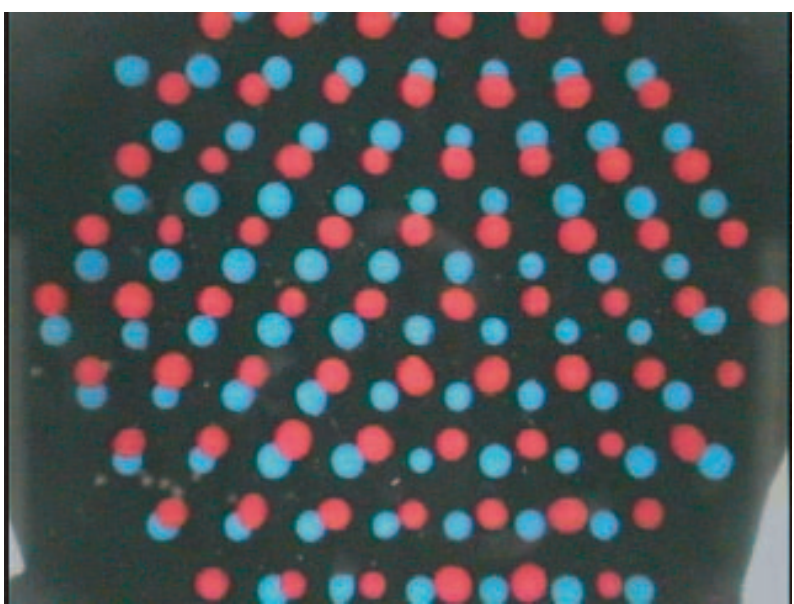

Fig. 3. Captured image by CCD camera

\section{EVALUATION EXPERIMENT}

\section{A. Measuring accuracy of movement vectors}

When a force vartical to the sensor surface is applied with a cylinder (5mm diameter), the observed displacement vectors of red markers are as shown in Fig.4. The magnitude of the force is $910 \mathrm{gf}$. Radial variation centered around force application point is generated, and observed displacement vectors is obtained by projection to $x-y$ plane. The lengths of the vectors in Fig.4 are five times as long as real displacement.

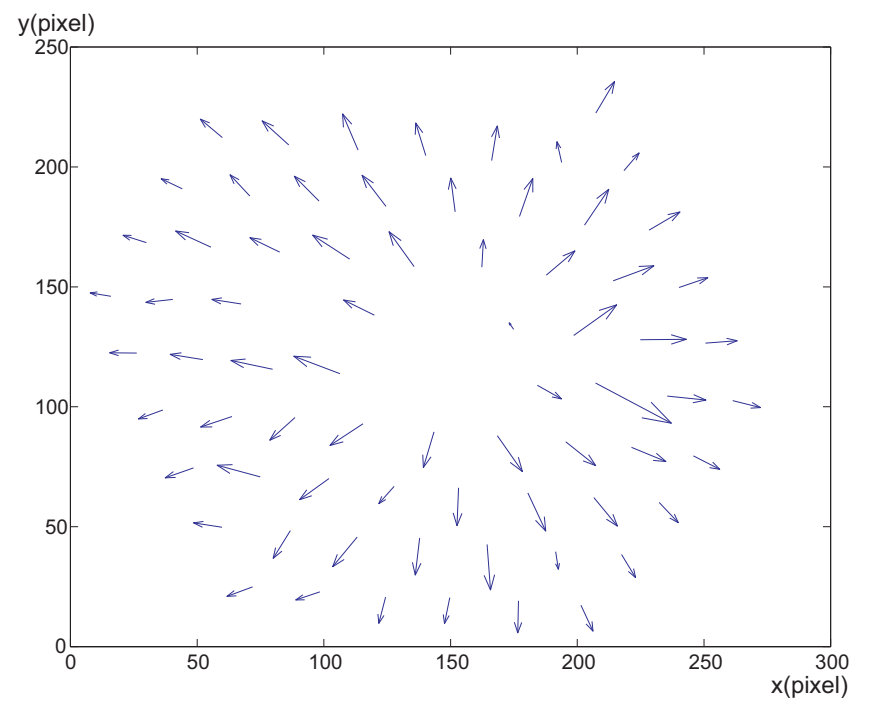

Fig. 4. Calculated displacement vectors of red markers when applied a $z$ directional force

As a first evaluation experiment, we investigated the accuracy of the above-mentioned method for displacement vector measurement. Two images of the markers are captured, the first when no force was applied, and the second which was displaced 3 pixels in the $x$-direction. Displacement vectors of the markers were calculated using these two images. The mean $x$ and $y$ displacements of red and blue markers are presented in Table 1. It is apparent that the displacements are properly 
measured, and with a standard deviation of less than 0.1 pixels, which corresponds to $50 \mu \mathrm{m}$, indicating considerable accuracy.

TABLE I

Test of Measuring Movememt

\begin{tabular}{|c|c|c|c|}
\hline Component & direction & Average(pixel) & SD \\
\hline \multirow{2}{*}{ Blue } & $+\mathrm{x}$ & 2.98 & 0.06 \\
\cline { 2 - 4 } & $+\mathrm{y}$ & 0.00 & 0.06 \\
\hline \multirow{2}{*}{ Red } & $+\mathrm{x}$ & 3.00 & 0.09 \\
\cline { 2 - 4 } & $+\mathrm{y}$ & 0.00 & 0.10 \\
\hline
\end{tabular}

\section{B. Evaluation of calculated force vectors}

Fig.5(a) shows the distribution of three dimensional force vectors when a force vertical to the surface was applied using a cylinder of $5 \mathrm{~mm}$ diameter, and Fig.5(b) shows the distribution of force parallel to the surface using a cylinder of $12 \mathrm{~mm}$. In this section, these measured force vectors are evaluated.

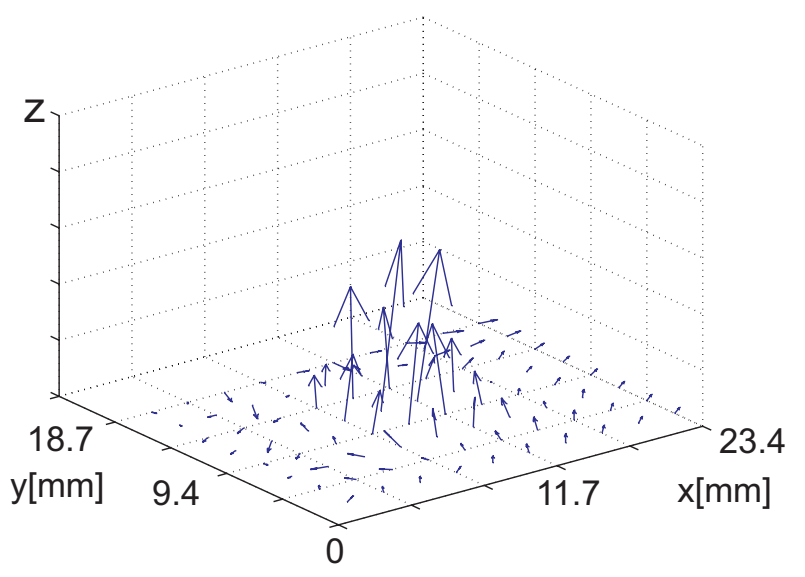

(a)Three dimensional force vectors when force is applied z-direction

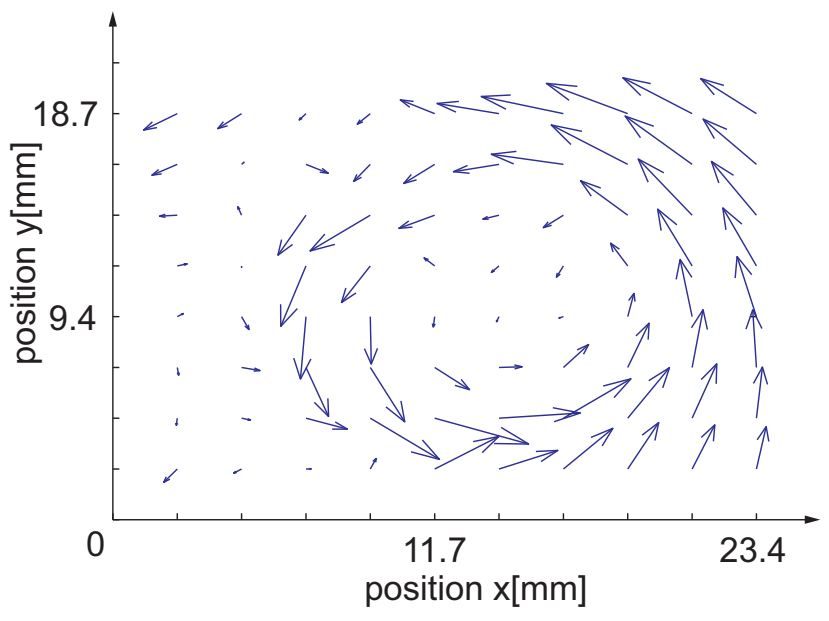

(b)force vectors parallel to the surface when torqued force is applied

Fig. 5. Measured distribution of force vectors
1) Effectiveness of two layers of markers: We simulate the effectiveness of the two layers of markers and the influence of distance between the blue and red layer.

Given a force distribution, displacement vectors are calculated using Eq.5. Marker positions of the simulation were derived from an actual CCD image. An error was added to these movement vectors with a mean and standard deviation of Opixel and 0.1pixel, respectively. Using the error added to the displacement vectors, we then calculate distribution of force. The effect of two layers is investigated by comparing between calculated and actual force vectors. This simulation is iterated as the given distance between the two layers is changed. The numerical value used in this evaluation is the mean difference between calculated and actual force obtained in each direction $x, y$, and $z$.

Number of sampling points is $10 \times 8$ points. A certain sampling point located at $x_{f}$ in the $x$-axis position and $y_{f}$ in the $y$-axis position is represented as $\left(x_{f}, y_{f}\right)$. The given force is equally applied on an area surrounded by the points $(4,3),(7,3),(4,6),(7,6)$, with a magnitude of $100 \mathrm{gf}$ in the $z$-direction and $50 \mathrm{gf}$ in the $y$-direction.

Fig.6 shows the difference between the actual force and the calculated force obtained from the simulation. The value of the horizontal axis means, starting from the left, the case of only the blue layer, the case of blue and red marker layer at the same depth, each case with a distance between the two layers from $1 \mathrm{~mm}$ to $6 \mathrm{~mm}$, then $8 \mathrm{~mm}$ and $13 \mathrm{~mm}$. The case that the blue and red layers are located at same depth is equivalent to the case that the density of sampling points at only one layer is doubled. Though the average of the difference is almost the same in the first two cases, the more the layers are separated, the less the mean of the difference becomes. However, this trend bottoms out, and with much more separation, the mean difference increases. This conclusion suggests that the difference between the two layers is preferably about $2 \mathrm{~mm}$ or $3 \mathrm{~mm}$, which was the distance adopted in our tactile sensor.

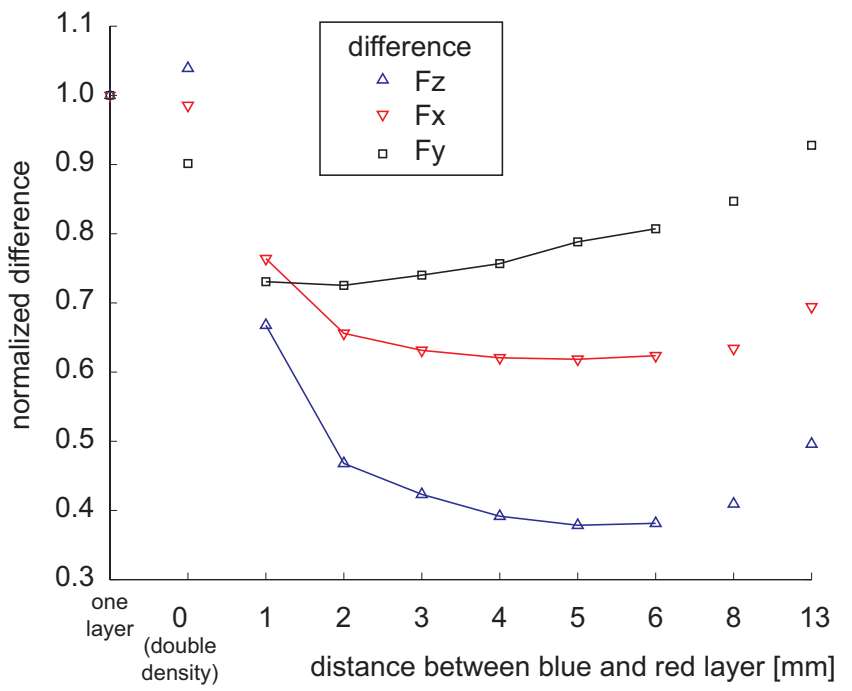

Fig. 6. Dependence of the error on the distance between two layers 
2) Resolution of magnitude and direction: In this section, we evaluate at first the resolution of the force magnitude, and then the resolution of direction. Each directional force $F_{x}, F_{y}$, $F_{z}$ is collected in 1-dimensional vector, and the summation of force is calculated as $F_{x}{ }^{\text {sum }}=\sum_{k=1}^{n m} F_{x}(k), F_{y}{ }^{\text {sum }}=$ $\sum_{k=1}^{n m} F_{y}(k), F_{z}{ }^{\text {sum }}=\sum_{k=1}^{n m} F_{z}(k)$. By comparing between this summation $F^{\text {sum }}$ and the actual applied force, we examine the resolution and linearity of measured force vectors. The actually applied force is measured by a 6-axis force sensor and controled by a $x y z$-stage.

First, we investigated magnitude of the measured force. Increasing the applied $z$-directional force from $0 \mathrm{gf}$ to $750 \mathrm{gf}$ by $50 \mathrm{gf}$ increments, the value acquired from the sensor is recorded, and this procedure is repeated 10 trials. The force is applied using a probe, the contact site of which formed $5 \mathrm{~mm}$ diameter hemisphere. Fig.7 shows the result of this experiment. This graph indicates that the sensor can sufficiently measure the force linearly and a magnitude resolution of about $30 \mathrm{gf}$. The elasticity of the sensor used in this experiment is 10 times harder than that of the human fingertip. By making the elasticity of the sensor as soft as the fingertip, the resolution improves to about $3 \mathrm{gf}$.

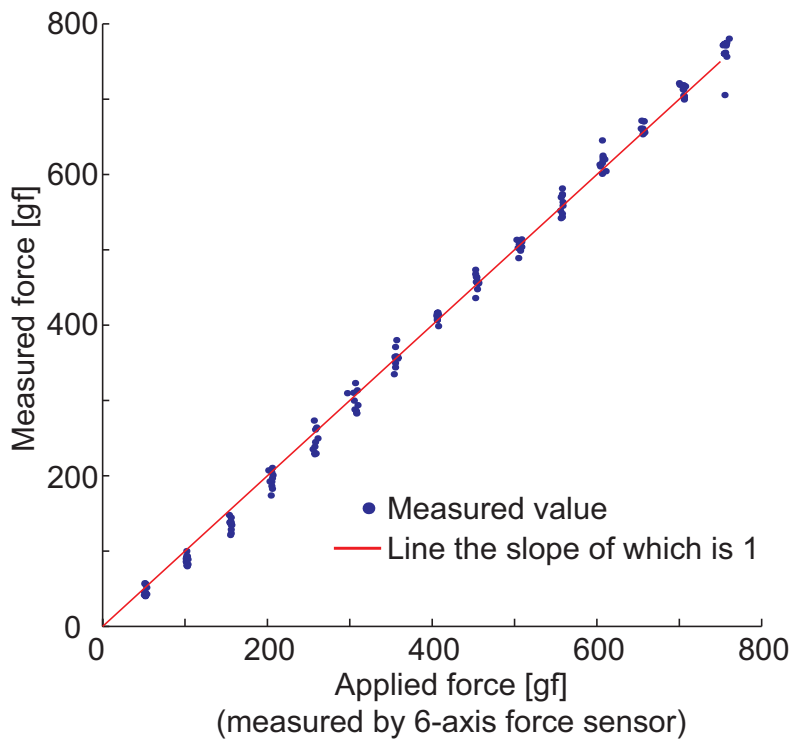

Fig. 7. Relation between applied and measured force of $z$-direction

Next, we estimated the resolution of angle of the force. The magnitude of the applied force is fixed at $400 \mathrm{gf}$, and the force is applied at an angle respective to the normal to the surface. This angle changes from $0 \mathrm{deg}$ to $45 \mathrm{deg}$ by $5 \mathrm{deg}$ increments. A cylinder with a diameter of $5 \mathrm{~mm}$ is used as a probe to apply force. The base plane of the probe is kept parallel to the $x y$-plane.

The experimental result is shown in Fig.8. It is apparentthat the proposed tactile sensor measures the angle of force precisely and linearly. The data spread of this graph shows that the resolution of the angle of measured force is about $5 \mathrm{deg}$.

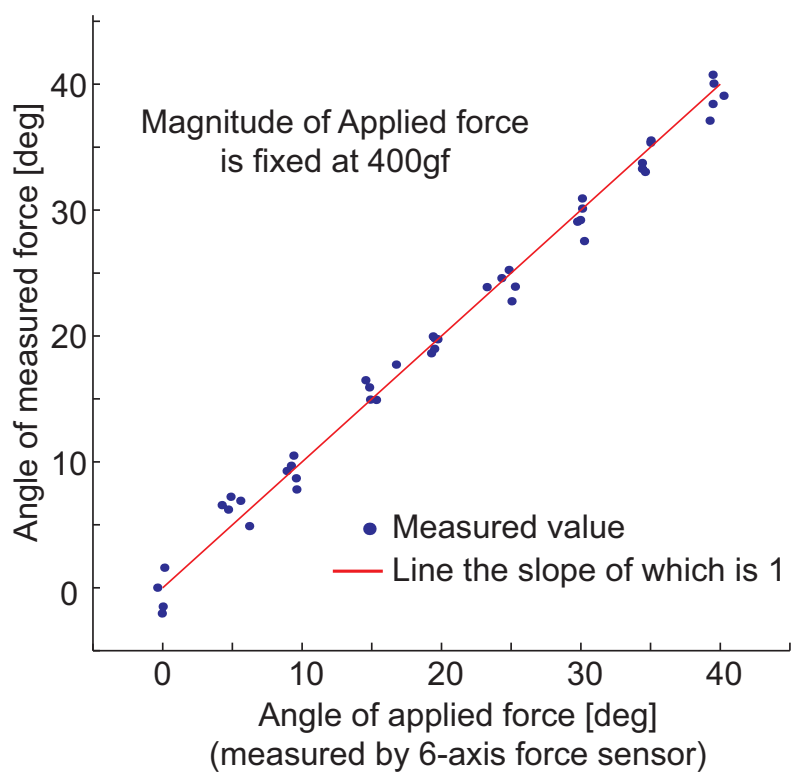

Fig. 8. Relation between applied and measured force with an angle from the surface

3) Spatical resolution of the distribution of forces: The proposed tactile sensor calculates force by using a pseudoinverse matrix, so the calculated result for each point includes an error and is distributed around its neighbors. Thus, in this section we investigate the effects of this error to the spatial resolution of the forces.

The evaluation uses a force distribution acquired by applying force at a certain point on the surface of the elastic body. However, the sampling interval is not fine enough to estimate the distributed error. Therefore we take a sequence explained as follows.

For simplicity, the force vector is assumed to be twodimensional. Two adjacent sampling points from the distribution of forces were selected. The distance between these two points was subdivided into $n$ points, and a given $z$-directional force was applied at each point. Then, the calculation of force vectors at each point was executed, with each point regarded as the origin. A graph is constructed with the horizontal axis the distance from each origin, and the vertical axis as the $z$ component of the calculated force vector.

By using the method explained above, the density of force sampling points is increased. Then, measuring the slope of the obtained force distribution led to the spatial resolution of force distribution.

Fig.9 shows the result of the experiment. The value of the vartical axis is normalized using the maximum value among the acquired data. The magnitude of applied force directed in the $z$-direction is fixed at $200 \mathrm{gf}$ and the adjacent sampling points are located at the center of the area in which force distribution is acquired. The actual sampling interval is $2.2 \mathrm{~mm}$ equal to that Fig.5 and theadjacent points are subdevided into $0.37 \mathrm{~mm}$ intervals. 
From the graph in Fig.9, it is apparent that the half value of width is about $4 \mathrm{~mm}$. That is to say, the spatial resolution of the forces is estimated at about $4 \mathrm{~mm}$. The obtained resolution is equal to one lead from the sampling interval, therefore the proposed sensor is proven to have sufficient spatial resolution.

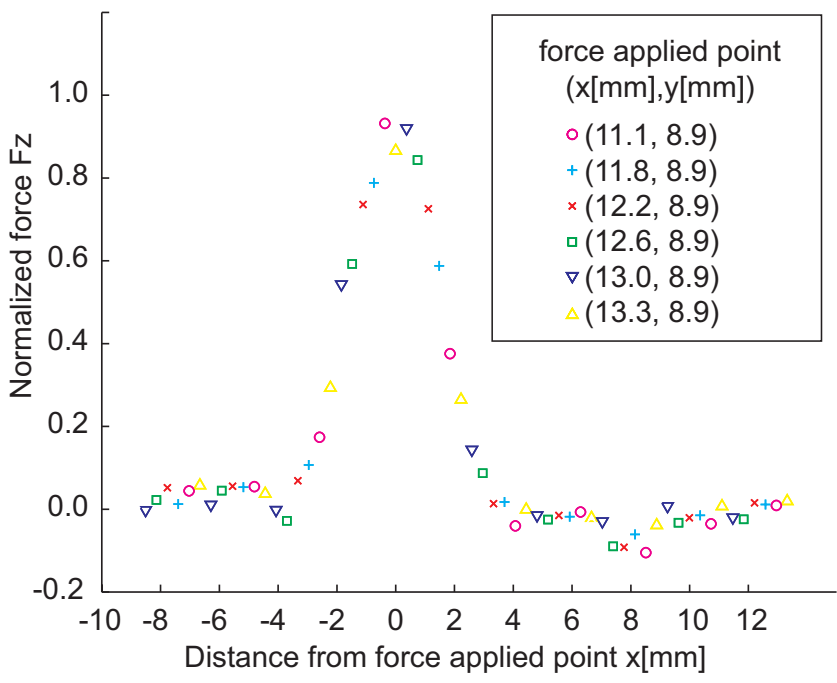

Fig. 9. $z$-component of measured force at high density sampling when point force is applied

\section{Estimation of Computational time}

In this section, we estimate computation time from the instant image data is loaded into main memory of the PC until the force vector data is obtained. For reference, the time from obtaining data until displaying this data like Fig.5 is also indicated. The computational time is acquired using timer based CPU frequency. The speed of the processor used in the PC for this benchmark, is $1.8 \mathrm{GHz}$ (Intel(R), Pentium4(R) processor) and the program runs under Windows 2000. Even though the fluctuation of the processor frequency was $100 \mathrm{MHz}$, this method had an accuracy of $10 \mu \mathrm{sec}$. Furthermore, by acquiring computation time more than once, the effects of other program interruptions can be decreased and the estimation is made more precise.

The computation time is measured 300 times and the average is shown in Table tab:time. It is apparent from this table that the computation time to obtain the force vectors is on the order of milliseconds and therefore suitable for real time applications.

TABLE II

COMPUTATION TIME UNTIL THE DATA OF FORCE VECTORS IS OBTAINED

\begin{tabular}{|c|c|c|}
\hline & time until obtain the forces & displaying time \\
\hline time[msec] & 2.9 & 1.9 \\
\hline
\end{tabular}

\section{Conclusion}

In this paper, we reported the evaluation of the proposed tactile sensor, which can measure the distribution of force vectors. The outcome of the experiments shows a $50 \mu \mathrm{m}$ accuracy of determining displacement vectors, a $30 \mathrm{gf}$ magnitude resolution, and a $5 \mathrm{deg}$ angle resolution of calculated force. The particular merit of this measurement principle of the sensor is that it is simple to photograph the markers in the elastic body; therefore it is easy to improve the sensitivity of the sensor by increasing the density of markers and changing the resolution of the color CCD camera.

Because of frequent contact to an object in practice, a tactile sensor must be durable. The proposed sensor is structured so that the CCD camera as an actual measurement device is separated from the contact point, in contrast to from a sensor in which sensing units are implanted. Hence, if the elastic portion of the sensor breaks, it is relatively easy to replace. Additionally, the tactile sensor we have developed can measure displacement vectors for an arbitrary shape, it can easily be mounted on a robot hand in the shape of a fingertip.

To append the human-like sense of touch to robots, a tactile sensor needs to measure distribution of force vectors, must be readily miniaturized, and capable of high-density measurement. Through our evaluation experiments, we have verified that the tactile sensor we have proposed fulfills these demands with high performance. At present, we are developing a finger shaped tactile sensor with the aim to mount it on a robot hand.

\section{REFERENCES}

[1] R.S.Fearing, "Using a Cylindrical Tactile Seosor for Determining Curvature", IEEE Trans. on Robotics and Automation, vol.7, No.6, pp.806$817,1991$.

[2] J. G. Silva, A. A. Carvalho, D. D. Silva, "A Strain Gage Tactile Sensor for Finger-Mounted Applications", IEEE Trans. on Instruction and Measurement, vol.51, No.1, pp.18-22, 1991.

[3] H. Shinoda, K. Matsumoto, S. Ando, "Acoustic Resonant Tensor Cell for Tactile Sensing", Proc. IEEE International Conference on Robotics and Automation, pp.3087-3092, 1997.

[4] D. T. V. Pawluk, J. S. Son, P. S. Wellman, W. J. Peine, R. D. Howe, "Distributed Pressure Sensor for Biomechanical Measurements", Journal of Biomechanical Engineering, Transactions of the ASME, Vol.120, No.2, pp. 302-305, 1998

[5] M.Ohoka, Y.Mituya, K.Hattori, I.Higashioka, "Data conversion capability of optical tactile sensor featuring an array of pyramidal projections", IEEE International Conference on Multisensor Fusion and Integration for Intelligent Systems, pp.573-580, 1996.

[6] K.Tanie, K.Komoriya, M. Kaneko, S.Tachi, "A HIGH RESOLUTION TACTILE SENSOR", Proceedings of the 4th International Conference on Robot Vision and Sensory Controls, pp.251-261, 1987.

[7] N. J. Ferrier, R. W. Brockett, "Reconstructing the Shape of a Deformable Membrane from Image Data", The International Journal of Robotics Research, Vol.19, No.9, pp.795-816, 2000.

[8] K. Kamiyama, H. Kajimoto, M. Inami, N. Kawakami, S.Tachi, "A Vision-based Tactile Sensor", The 11th International Conference on Artificial reality and Telexistence, 2001.

[9] L. D. Landau, E. M. Lifshitz, "Theory of Elasticity", BUTTERWORTHHEINEMANN, 1985.

[10] W. Menke, "Geophysical Data Analysis: Discrete Inverse Theory", Academic Press, Inc., 1989.

[11] R. S. Fearing, J. M. Hollerbach, "Basic Solid Mechanics for Tactile Sensing", The International Journal of Robotics Research, Vol.4,No.3,pp.40-54, 1985. 